KHTP-93-01

SNUCTP-93-05

\title{
Conformal Turbulence with Boundary
}

\author{
B.K. Chung, Soonkeon Nam, Q-Han Park ${ }^{\dagger}$ and H.J. Shin ${ }^{\ddagger}$ \\ Department of Physics \\ Kyung Hee University \\ Seoul, 130-701, Korea
}

\begin{abstract}
Based upon the formalism of conformal field theory with a boundary, we give a description of the boundary effect on fully developed two dimensional turbulence. Exact one and two point velocity correlation functions and energy power spectrum confined in the upper half plane are obtained using the image method. This result enables us to address the infrared problem of the theory of conformal turbulence.
\end{abstract}

\footnotetext{
^ E-mail address: nam@nms.kyunghee.ac.kr

$\dagger$ E-mail address: qpark@nms.kyunghee.ac.kr

$\ddagger$ E-mail address: shin@SHIN.kyunghee.ac.kr
} 
Turbulence arises in many physical systems, which obey simple but nonlinear equations of motions. ${ }^{[1]}$ The statistical nature of turbulence together with its nonlinear, nonequilibrium structures seem to require certain non-unitary quantum field theories for the theory of turbulence..$^{[2]}$ The appearance of divergences in convensional perturbative approach ${ }^{[3]}$ suggest the use of renormalization group technique. However, so far this has been unsuccessful leaving turbulence still an outstanding problem in theoretical physics.

Recently, Polyakov ${ }^{[4,5]}$ has proposed a new approach based on the assumption of conformal invariance in the inertial range of fully developed two dimensional turbulence. He argued that certain non-unitary conformal field theories (CFT's) provide exact solutions of the fundamental equations of turbulence, which are consistent with the known energy spectrum. ${ }^{[6]}$ However, Polyakov's solutions in terms of correlators of non-unitary CFT are plagued with infrared(IR) divergences and also highly dependent on the arbitrary vacuum expectation values of operators in the theory. Instead of taking the IR problem as a weakness of his approach, Polyakov $^{[4,5]}$ has conjectured that the resolution of the problem is actually the most important part of the theory.

In this letter, we introduce a physical boundary to a turbulent system in order to regulate the IR divergence. Using an 'image method', we determine exact one and two point velocity correlation functions and energy power spectrum which depend on the distance from the boundary. Away from the boundary, these functions quickly become isotropic which is in accordance with the Kolmogorov's local isotropy assumption. ${ }^{[7,2]}$ This allows us to use the boundary not only for handling the IR divergences, but also for an effective tool to understand the IR behavior of the theory. We attempt to give a physical interpretation to these results.

In two dimensions, the fluid motion is governed by the Navier-Stokes equation:

$$
\dot{\omega}+\epsilon_{\alpha \beta} \partial_{\alpha} \psi \partial_{\beta} \partial^{2} \psi=\nu \partial^{2} \omega+\text { stirring force, } \quad(\alpha, \beta=1,2)
$$

where $\omega$ and $\psi$ denote the vorticity and the stream function respectively and $\nu$ 
is the viscosity. Stream function is related to the vorticity and velocity through $\omega=\partial^{2} \psi$ and $v_{\alpha}=\epsilon_{\alpha \beta} \partial_{\beta} \psi$. A typical stirring force is provided by letting fluid flow through a grid consisting of very thin bars. For fluid with a sufficiently large Reynolds number, fully developed turbulence emerges 30 to 40 times the grid bar spacing away, where the stirring force is absent. ${ }^{[2]}$ The boundary which we use in this paper is parallel to the direction of the incoming flow.

In the following, we first review the case without boundary. Polyakov's CFT approach is to identify the stream function $\psi$ with a primary field of a certain minimal CFT. In the static and inviscid case, the Navier-Stokes equation reduces to " $\epsilon_{\alpha \beta} \partial_{\alpha} \psi \partial_{\beta} \partial^{2} \psi$ " $=0$. This equation has meaning only when we exercise care in defining the operator product at the coinciding points, e.g., by using the point splitting method,

$$
\text { " } \epsilon_{\alpha \beta} \partial_{\alpha} \psi(z) \partial_{\beta} \partial^{2} \psi(z) "=\lim _{a \rightarrow 0} \epsilon_{\alpha \beta} \partial_{\alpha} \psi(z+a) \partial_{\beta} \partial^{2} \psi(z),
$$

where lim implies angle averaging. In CFT, the operator product expansion (OPE) of $\psi$ has the following structure:

$$
\psi(z+a) \psi(z)=(a \bar{a})^{\Delta_{\phi}-2 \Delta_{\psi}}\{\phi(z)+\text { descendents }\}+\cdots,
$$

where $\phi$ is the minimal dimension operator of dimension $\Delta_{\phi}$ which is most relevant as $a \rightarrow 0$. This leads to

$$
" \epsilon_{\alpha \beta} \partial_{\alpha} \psi(z) \partial_{\beta} \partial^{2} \psi(z) " \sim \lim _{a \rightarrow 0}(a \bar{a})^{\Delta_{\phi}-2 \Delta_{\psi}}\left[L_{-2} \bar{L}_{-1}^{2}-\bar{L}_{-2} L_{-1}^{2}\right] \phi(z),
$$

with $L_{-n}$ being Virasoro generators. The right hand side of Eq.(4) vanishes, i.e., the Navier-Stokes equation is satisfied, for the following two cases: First is when $\left[L_{-2} \bar{L}_{-1}^{2}-\bar{L}_{-2} L_{-1}^{2}\right] \phi=0$, which for example is satified when $\phi$ is degenerate on the level two. The simplest concrete example of this is the minimal model of $p=2, q=5$, which we denote $\mathcal{M}_{(2,5)}$, the critical Yang-Lee edge singularity. ${ }^{[8]}$ 
Second case is when $\Delta_{\phi}>2 \Delta_{\psi}$. This together with a further restriction coming from the constant enstrophy flux condition ${ }^{[9]}$ has led Polyakov and others find series of exact solutions of conformal turbulence. ${ }^{[4,5,10-13]}$ The first case of $\mathcal{M}_{(2,5)}$ does not have constant enstrophy flux, thus requires a subtle balance between vorticity and energy injection. ${ }^{[4]}$

In order to calculate the energy spectrum $E(k)$, we need the two point function of $\psi$;

$$
\left\langle\psi\left(z_{1}\right) \psi\left(z_{2}\right)\right\rangle \sim \frac{\langle I\rangle}{\left|z_{1}-z_{2}\right|^{4 \Delta_{\psi}}}+\frac{\left\langle\phi\left(z_{2}\right)\right\rangle}{\left|z_{1}-z_{2}\right|^{4 \Delta_{\psi}-2 \Delta_{\phi}}}+\cdots,
$$

which results in the power law behavior

$$
E(k) \sim k^{\alpha},
$$

where $\alpha=4 \Delta_{\psi}+1$ for $\langle\phi\rangle=0$, and $\alpha=4 \Delta_{\psi}-2 \Delta_{\phi}+1$ for $\langle\phi\rangle \neq 0$. Unlike in unitary cases, one point function $\langle\phi\rangle$ is not necessarily zero in non-unitary theories, and as we will see in the following, $\langle\phi\rangle$ can be determined by boundary condition.

In this letter, we will focus on $\mathcal{M}_{(2,5)}$ minimal model restricted to the upper half plane $(y>0)$ to investigate boundary effects on fully developed turbulence explicitly. One way to see the effect of a boundary on a turbulent flow would be to imagine that each eddy in turbulent flow has an image eddy on the other side of the boundary, similar to image charges of electrostatics near a conductor. This is consistent with the fact that vertical component of fluid velocity vector vanishes at the boundary. Furthermore, this picture enables us to extend Polyakov's CFT approach to turbulence to the case with boundary.

Cardy ${ }^{[14]}$ introduced CFT with boundary utilizing the image method to describe surface critical behavior in two dimensions. He has shown that the restricted conformal transformation which preserves boundary conditions can be achieved by extending the energy momentum tensor $T(z)$ analytically in the lower half plane through $T(z)=\bar{T}(\bar{z})$, when $y=\operatorname{Im} z<0$. One consequence of it is that for the degenerate conformal field theories, the $n$-point function $\left\langle\phi\left(z_{1}, \bar{z}_{1}\right) \cdots \phi\left(z_{n}, \bar{z}_{n}\right)\right\rangle_{b}$ 
can be obtained systematically from the bulk $2 n$-point function, where $\langle\cdots\rangle_{b}$ denotes correlation functions in the upper half plane. This is so because the $n$-point function $\left\langle\phi\left(z_{1}, \bar{z}_{1}\right) \cdots \phi\left(z_{n}, \bar{z}_{n}\right)\right\rangle_{b}$ satisfies the same differential equation as does the bulk $2 n$ point function consisting of charges in the upper half plane as well as their images in the lower half plane.

$\mathcal{M}_{(2,5)}$ minimal model has only one nontrivial primary field $\phi_{(1,2)}$, of conformal dimension $\Delta_{(1,2)}=-1 / 5$, whose normalization is such that $\left\langle\phi_{(1,2)}\left(z_{1}\right) \phi_{(1,2)}\left(z_{2}\right)\right\rangle=$ $\left|z_{1}-z_{2}\right|^{4 / 5}$. Here we identify $i$ times the primary field $\phi_{(1,2)}$ as the stream function $\psi$. The factor $i$ is required for the following reason; if we neglect the factor $i$, we can not simultaneously have a real one point function and a positive two point function of velocity, which are necessary in order to be physical.

Following the Cardy's image method, we obtain the one point function of $\psi$ with boundary from a two point function of $\psi(z)$ and its image $\psi(\bar{z})$ across the boundary such that

$$
\langle\psi(z, \bar{z})\rangle_{b}=\langle\psi(x, y)\rangle_{b}=d_{\psi} y^{2 / 5},(z=x+i y, y>0)
$$

where $d_{\psi}$ is an arbitrary real constant. In the unitary case with boundary, $d_{\psi}$ is fixed by the asymptotic behavior of two point fuction: $\left\langle\psi\left(x_{1}, y_{1}\right) \psi\left(x_{2}, y_{2}\right)\right\rangle_{b} \rightarrow$ $\left\langle\psi\left(y_{1}\right)\right\rangle_{b}\left\langle\psi\left(y_{2}\right)\right\rangle_{b}$ as $\left|x_{1}-x_{2}\right| \rightarrow \infty$, whereas in the non-unitary case $d_{\psi}$ is in general arbitrary. The mean velocity can be obtained from the one point function of $\psi$ such that

$$
\begin{aligned}
\left\langle v_{x}(x, y)\right\rangle_{b} & =\left\langle\partial_{y} \psi(x, y)\right\rangle_{b}=\frac{2}{5} d_{\psi} y^{-3 / 5} \\
\left\langle v_{y}(x, y)\right\rangle_{b} & =-\left\langle\partial_{x} \psi(x, y)\right\rangle_{b}=0
\end{aligned}
$$

We interpret this result as describing a system with a net drift velocity profile of turbulent flow parallel to the boundary. The drift velocity in Eq.(8) goes to infinity as $y \rightarrow 0$, so it should be rounded off over a certain distance from the boundary.

In order to calculate the energy spectrum of the system, we need the two point function of velocity. Again, this can be obtained from the bulk four point 
function of $\psi$ involving two in the upper half plane and two images in the lower half. For $\mathcal{M}_{(2,5)}$, which is degenerate on the level two, the bulk four point function satisfies a second order differential equation. Solutions of the differential equation can be put in terms of linear combinations of hypergeometric functions of variable $\xi=-\frac{\left|z_{1}-z_{2}\right|^{2}}{4 y_{1} y_{2}}:$

$$
\left\langle\psi\left(z_{1}, \bar{z}_{1}\right) \psi\left(z_{2}, \bar{z}_{2}\right)\right\rangle_{b}=\left(\frac{z_{13} z_{24}}{z_{12} z_{23} z_{34} z_{14}}\right)^{-2 / 5}\left(a F_{1}(\xi)+b F_{2}(\xi)\right)
$$

where we use the notation $z_{3}=\bar{z}_{1}, z_{4}=\bar{z}_{2}, z_{i j}=z_{i}-z_{j}$, and $F_{1}={ }_{2} F_{1}\left(\frac{3}{5}, \frac{4}{5} ; \frac{6}{5} ; \xi\right)$, $F_{2}=\xi^{-1 / 5}{ }_{2} F_{1}\left(\frac{3}{5}, \frac{2}{5} ; \frac{4}{5} ; \xi\right)$. $a$ and $b$ are constants which can be determined by looking at the limiting behavior of the correlator as $\left|z_{1}-z_{2}\right| \rightarrow 0$. In this limit, the right hand side of Eq.(9) becomes

$$
\left\langle\psi\left(z_{1}, \bar{z}_{1}\right) \psi\left(z_{2}, \bar{z}_{2}\right)\right\rangle_{b} \rightarrow\left|z_{1}-z_{2}\right|^{4 / 5}\left[a+b\left(-\frac{\left|z_{1}-z_{2}\right|^{2}}{4 y^{2}}\right)^{-1 / 5}\right]
$$

where $y=\left(y_{1}+y_{2}\right) / 2$. On the other hand, the OPE of $\psi\left(z_{1}\right) \psi\left(z_{2}\right)$ as $z_{1} \rightarrow z_{2}$ gives

$\frac{C_{\psi \psi I}}{\left|z_{1}-z_{2}\right|^{4 \Delta_{\psi}}}\langle I\rangle_{b}+\frac{C_{\psi \psi \psi}}{\left|z_{1}-z_{2}\right|^{4 \Delta_{\psi}-2 \Delta_{\psi}}}\langle\psi\rangle_{b}=-\left|z_{1}-z_{2}\right|^{4 / 5}+\left|z_{1}-z_{2}\right|^{2 / 5} C_{\psi \psi \psi} d_{\psi} y^{2 / 5}$.

$C_{\psi \psi I}$ and $C_{\psi \psi \psi}$ are structure functions which are determined from the three point function of the bulk theory. Explicit calculation shows that $C_{\psi \psi I}=-1$ and $C_{\psi \psi \psi}=|C|^{1 / 2}$, where $C=-\frac{\Gamma(6 / 5)^{2} \Gamma(1 / 5) \Gamma(2 / 5)}{\Gamma(3 / 5) \Gamma(4 / 5)^{3}} \cdot{ }^{[8]}$ Comparing Eq.(10) and Eq.(11), we get $a=-1$ and $b=2^{-2 / 5} d_{\psi}|C|^{1 / 2}$.

The correlators of velocity components can be obtained by differentiating the stream function correlator, for example, $\left\langle v_{y}\left(z_{1}\right) v_{y}\left(z_{2}\right)\right\rangle_{b}=\partial_{x_{1}} \partial_{x_{2}}\left\langle\psi\left(z_{1}\right) \psi\left(z_{2}\right)\right\rangle_{b}$. In order to obtain the energy spectrum $E(k)$ for $k \gg k_{0}$ where $k_{0}$ is the characteristic input wavenumber, we consider the form of velocity correlators in the small distance 
limit. When $x_{1}=x_{2}$ and $y_{1}=y+\epsilon / 2, y_{2}=y-\epsilon / 2(\epsilon \ll y)$, the velocity correlator is given by

$$
\begin{aligned}
\left\langle v_{y}\left(x_{1}, y_{1}\right) v_{y}\left(x_{2}, y_{2}\right)\right\rangle_{b} & =\frac{4}{5} \epsilon^{-6 / 5}\left(1-\frac{3}{440} \frac{\epsilon^{4}}{y^{4}}-\frac{7}{7040} \frac{\epsilon^{6}}{y^{6}}+\cdots\right) \\
+ & \frac{2}{5} d_{\psi}|C|^{1 / 2} \epsilon^{-8 / 5} y^{2 / 5}\left(1+\frac{1}{10} \frac{\epsilon^{2}}{y^{2}}-\frac{7}{600} \frac{\epsilon^{4}}{y^{4}}-\frac{3}{2000} \frac{\epsilon^{6}}{y^{6}}+\cdots\right)
\end{aligned}
$$

When the mean velocity is zero $\left(d_{\psi}=0\right)$ and when moved far away from the boundary, i.e., when $y \gg 1$, the isotropic bulk two point velocity correlation function is recovered. Similarly, we have

$$
\begin{gathered}
\left\langle v_{x}\left(x_{1}, y_{1}\right) v_{x}\left(x_{2}, y_{2}\right)\right\rangle_{b}=-\frac{4}{25} \epsilon^{-6 / 5}\left(1+\frac{57}{440} \frac{\epsilon^{4}}{y^{4}}+\frac{243}{7040} \frac{\epsilon^{6}}{y^{6}}+\cdots\right) \\
-\frac{6}{25} d_{\psi}|C|^{1 / 2} \epsilon^{-8 / 5} y^{2 / 5}\left(1+\frac{1}{10} \frac{\epsilon^{2}}{y^{2}}+\frac{179}{1800} \frac{\epsilon^{4}}{y^{4}}+\frac{47}{2000} \frac{\epsilon^{6}}{y^{6}}+\cdots\right)
\end{gathered}
$$

and also $\left\langle v_{x}\left(x_{1}, y_{1}\right) v_{y}\left(x_{2}, y_{2}\right)\right\rangle_{b}=0$. It is straighforward to show that the leading order terms in Eq.(12) and Eq.(13) are isotropic in the sense that they do not depend on the limiting procedure of $\left|z_{1}-z_{2}\right| \rightarrow 0$.

The energy density at point $(x, y)$ is given by $\frac{1}{2}\left\langle v_{\alpha}^{2}(x, y)\right\rangle_{b}=\int d k E_{(x, y)}(k)$. In the momentum space, the energy spectrum $E_{(x, y)}(k)$ is given in terms of velocity correlators:

$$
E_{(x, y)}(k) \sim \frac{1}{8 \pi^{2}} \int d^{2} x^{\prime} e^{i k_{\alpha}\left(x_{\alpha}-x_{\alpha}^{\prime}\right)}\left\langle v_{\beta}\left(x^{\prime}, y^{\prime}\right) v_{\beta}(x, y)\right\rangle_{b}
$$

In the homogeneous case, $E_{(x, y)}(k)$ does not depend on the position $(x, y)$ which, however in the presence of boundary, is a function of the position. From Eqs.(12)(14), we get

$$
E_{(x, y)}(k) \sim \pi\left(A k^{1 / 5}+B d_{\psi} y^{2 / 5} k^{3 / 5}\right)
$$

where $A=2^{-1 / 5} \frac{16}{25} \frac{\Gamma(2 / 5)}{\Gamma(3 / 5)}$ and $B=2^{-3 / 5} \frac{4}{25}|C|^{1 / 2} \frac{\Gamma(1 / 5)}{\Gamma(4 / 5)}$. It is remarkable that the boundary effect explicitly shows up through the one point function of the 
stream function $\langle\psi(z)\rangle_{b}=d_{\psi} y^{2 / 5}$. In general, when $\langle\phi\rangle$ in Eq.(5) does not vanish, Polyakov argued that the energy spectrum is described by $E(k) \sim k^{4 \Delta_{\psi}-2 \Delta_{\phi}+1}$ while for $\langle\phi\rangle_{b}=0, E(k) \sim k^{4 \Delta_{\psi}+1}$. Above two cases emerge in a unified form in our approach, and from this we see that the spectrum near the boundary is dominated by $E(k) \sim k^{4 \Delta_{\psi}+1}$ and as the distance from the boundary increases, the $E(k) \sim k^{4 \Delta_{\psi}-2 \Delta_{\phi}+1}$ behavior becomes more and more dominant. This is one of our main result, which seems to be a salient boundary effect in any CFT approach to turbulence. It would be very interesting to verify such a phenomena experimentally in real turbulent systems.

One of the basic assumptions in turbulence theory is the Kolmogorov's idea of local isotropy, that is, small-scale disturbances in turbulence with sufficiently large Reynolds number can be regarded as isotropic, regardless of large scale anisotropy. This is consistent with our CFT approach. In our case, large scale anisotropy is introduced through the boundary. Nevertheless small scale fluctuations become isotropic quickly as we move away from the boundary. In order to see this explicitly, we consider the velocity two point function of Eq.(12) rotated by 90 degrees with respect to the boundary. This is given by

$$
\begin{gathered}
\left\langle v_{x}\left(x_{1}, y_{1}\right) v_{x}\left(x_{2}, y_{2}\right)\right\rangle_{b}=\frac{4}{5} \epsilon^{-6 / 5}\left(1-\frac{3}{440} \frac{\epsilon^{4}}{y^{4}}+\frac{57}{7040} \frac{\epsilon^{6}}{y^{6}}+\cdots\right) \\
+\frac{2}{5} d_{\psi}|C|^{1 / 2} \epsilon^{-8 / 5} y^{2 / 5}\left(1+\frac{1}{20} \frac{\epsilon^{2}}{y^{2}}-\frac{23}{300} \frac{\epsilon^{4}}{y^{4}}+\frac{9}{250} \frac{\epsilon^{6}}{y^{6}}+\cdots\right)
\end{gathered}
$$

where the limit is now such that $y_{1}=y_{2}=y$ and $x_{1}=x_{2}+\epsilon$. Comparing this with Eq.(12), we see that the isotropy is broken only at the sixth order of $\epsilon / y$ in $d_{\psi}$ independent terms, and at the second order in $d_{\psi}$ dependent terms.

In this letter, we have determined one and two point velocity correlation functions by introducing a boundary, which incorporates the large scale structure into the scheme of CFT approach to turbulence. In his application of CFT to the turbulence problem, Polyakov ${ }^{[4,5]}$ introduced an IR cut-off by adding $\delta$ functions in the momentum space. In our approach, a more realistic IR cut-off is introduced 
through a boundary. Although our model defined on a half plane still has an IR divergence problem, this can easily be remedied by employing more complicated boundaries. Details about other types of boundary and also different minimal CFT's leading to more realistic cases will be considered elsewhere.

\section{Acknowledgements}

We would like to thank Yup Kim for discussions. This work was supported

in part by the program of Basic Science Research, Ministry of Education, and by Korea Science and Engineering Foundation. 


\section{REFERENCES}

1. See for example, L.D. Landau and E.M. Lifshitz, Fluid Mechanics, Pergamon, Oxford (1984).

2. A.S. Monin and A.M. Yaglom, Statistical Fluid Mechanics, The MIT Press, Cambridge (1975).

3. A.A. Migdal, Mod. Phys. Lett. A6 (1991) 1023.

4. A.M. Polyakov, Princeton Univ. preprint PUPT-1341, hep-th/9209046.

5. A.M. Polyakov, Princeton Univ. preprint PUPT-1369, hep-th/9212145.

6. B. Legras, P. Santangelo, and R. Benzi, Europhys. Lett. 5 (1988) 37.

7. A.N. Kolmogorov, J. Fluid Mech. 13 (1962) 82.

8. J.L. Cardy, Phys. Rev. Lett. 54 (1985) 1354.

9. R. Kraichnan, Phys. Fluid, 10 (1967) 1417.

10. G. Ferretti and Z. Yang, Rochester preprint UR-1296, hep-th/9212021.

11. D.A. Lowe, Princeton University preprint PUPT-1362, hep-th/9212019.

12. G. Falkovich and A. Hanany, WIS-92/88/Nov-PH, hep-th/9212015.

13. Y. Matsuo, Univ. of Tokyo preprint UT-620, hep-th/9212010.

14. J.L. Cardy, Nucl. Phys. B240 [FS12] (1984) 514. 